\title{
Sleep disorder in HIV/AIDS Patients
}

\author{
Desak Ketut Indrasari Utami aa*, Astari Arum Cendani Goler ${ }^{\mathrm{b}}$, \\ aindrasari@unud.ac.id
}

${ }^{a}$ Neurologist consultant in Department of Neurology, Faculty of Medicine Udayana University, Denpasar, Bali 80113, Indonesia

${ }^{\mathrm{b}}$ Resident Neurology, Department of Neurology, Faculty of Medicine Udayana University, Denpasar, Bali 80113, Indonesia

\begin{abstract}
Human Immunodeficiency Virus (HIV) contamination has been perceived as one of the genuine medical issues that affect society, culture, economy and governmental issues. From years instances of HIV/AIDS in Indonesia keeps on expanding. In light of information from Pusdatin in 2020, it was accounted for that during the most recent eleven years the quantity of HIV cases in Indonesia arrived at its top in 2019, which was 50,282 cases. Patients living with HIV/AIDS currently face new difficulties in living for a really long time with conditions related with ongoing sicknesses. Existing investigations show that somewhere in the range of 30 and $73 \%$ of the HIV seropositive populace gripe of helpless sleep quality. The utilization of the medication evafirenz and financial issues can disrupt sleep term causing diminished consideration the following day. Sleep deprivation is accounted for as the most well-known sort of sleep problem in patients with HIV/AIDS. Other sleep issues incorporate obstructive sleep apnea condition, parasomnias, sleepless leg disorder, and circadian cadence sleep problems.
\end{abstract}

Keywords: Sleep disorders, HIV, sleep quality.

\section{Introduction}

Sleep is one of the functions of life. Various medical disorders can affect sleep function and disruption of sleep patterns themselves can affect the quality of human life and even interfere with health that can be life-threatening. health status or illness (Taibi, 2013). Sleep quality disorders usually occur in people with psychological disorders, physiological disorders and including people with HIV. Sleep quality disorders that occur in patients with HIV can affect the quality of life and health status (Handayani et al., 2019).

As per Pusdatin information in 2020, it was accounted for that during the most recent eleven years the quantity of HIV cases in Indonesia arrived at its top in 2019, which was 50,282 cases. Information got from the WHO in 2019, viewed as $78 \%$ of new HIV contaminations in the populace in the Asia Pacific district. Helps cases were viewed as the most noteworthy during the most recent eleven years in 2013, to be specific 12,214 cases. The AIDS Case Fatality Rate in Indonesia from 2005 to $2019 \mathrm{kept}$ on declining. One reason for this decrease is that Indonesia's AIDS treatment endeavors have been critical and have prevailed with regards to lessening the quantity of passings from AID (Indonesian Ministry of Health, 2020)

Sleep aggravations are a typical issue and have been accounted for in $10 \%$ to $40 \%$ of everybody. These issues can influence personal satisfaction, physical and social working and can likewise prompt persistent exhaustion. Individuals with HIV are more inclined to sleep aggravations. In light of the Pittsburgh Sleep Quality Index, it has been accounted for that $73 \%$ to 100 percent of HIV positive individuals experience fluctuating levels of sleep issues. Ordinary sleep engineering can be changed and slow wave sleep is essentially expanded in HIV patients. Bigger sleep beginning inactivity, early arousing, continuous evening time enlightenments, and decreased sleep productivity have been accounted for. Nervousness and wretchedness are mental variables that influence sleep quality. Sleep aggravations are a typical manifestation of melancholy. Patients with tension problems experience deferred sleep beginning and decreased all out sleep time. HIVpositive patients with more elevated levels of gloom or uneasiness showed more sleep aggravations than HIV-tainted people without indications of sorrow and nervousness. Actual indications, for example, torment, stomach cramps, looseness of the bowels, incontinence, tingling, consuming, fever, night sweats, hack, and dyspnea can likewise cause sleep unsettling influences in individuals with HIV (Oshinaike et al., 2014).

\section{Sleep Disorders in HIV}

Sleep is defined as a physiological and recurrent form of reversible loss of consciousness in which there is a global decline in cognitive function so that the brain does not respond fully to surrounding stimuli (Alikhani et al., 2020).

Human immunodeficiency virus (HIV) is a chronic infectious disease that attacks the patient's immune system. Most people with HIV can experience complications such as sleep disturbances, anxiety, depression, and socio-economic impacts. As the disease progresses, adequate therapy is urgently needed (Schank et al., 2021) 
Sleep disturbances in people living with HIV is currently unclear, where this condition is thought to be related to CD4 cell count, HIV RNA viral load, social problems or ARV treatment. ${ }^{1,6}$ Improvements in medical care and available therapies such as combination antiretroviral therapy for people with HIV with HIV/AIDS has significantly reduced morbidity and mortality. Pathophysiology ARV drugs (Efavirenz) have side effects, one of which is sleep disturbance. High plasma concentrations of efavirenz will increase the incidence of neuropsychiatric side effects that have the ability to penetrate the blood brain barrier. High concentrations in plasma cause side effects such as sleep disturbances, nightmares, difficulty concentrating, dizziness, insomnia, and skin rashes. Therefore consumption of efavirenz is recommended at bedtime to minimize neuropsychiatric side effects. It is well absorbed by oral administration. Efavirenz is effective in suppressing plasma viral load but there have been many reports of neuropsychiatric side effects following efavirenz use. on the other hand. Poor quality of life can affect sleep quality, the degeneration process causes effective sleep time to decrease, and causes inadequate sleep quality to be achieved and causes various kinds of sleep complaints that can interfere with quality of life (Bandera et al., 2019).

Sleep unsettling influences that regularly seem can be exacerbated by uneasiness issues, financial conditions, disgrace, discouragement, self-destructive ideation and conditions that can never again work. Right now, the occurrence of sleep deprivation in HIV patients is as yet being examined, yet a few investigations have shown that HIV sickness movement influences the focal sensory system, artful contaminations of the focal sensory system, pharmacological impacts of antiretroviral drugs (efavirenz), emotional wellness and chronic drug use in HIV patients. counting a background marked by alcohol drunk (Alikhani et al., 2020; Gutierrez et al., 2019). Oshinaike et al, 2014 detailed a high predominance of helpless sleep quality $(58.9 \%)$ in HIV-contaminated patients in Nigeria. Helpless sleep quality was related with higher PSQI scores in many parts, showing the intricacy of the issue. Beforehand, Wu et al. revealed that the pervasiveness of helpless sleep quality in HIV-contaminated patients went from $40 \%$ to 100 percent. This distinction in commonness might be because of an assortment of variables remembering contrasts for geographic region, member attributes, and estimation instruments between studies in mexico, The predominance of helpless sleep quality in everyone goes from $21 \%$ to $26 \%$ (Oshinaike et al., 2014).

Helpless sleep quality was anticipated by burdensome side effects, utilization of illegal medications, CD4 <200 cells $/ \mu \mathrm{L}$, and longer time since HIV conclusion related with changes in the immunoinflammatory framework found in HIVtainted patients. Adjusted provocative reaction is related with HIV contamination, even in those with imperceptible viral burdens. CNS irritation is optional to HIV proteins (eg Tat protein), albeit dynamic viral replication is missing. Expanded coursing proinflammatory cytokines change action in a few cerebrum areas that influence sleep work, including the autonomic administrative focus, nerve center, limbic framework, mesolimbic cortex, and brainstem cores. The connection among aggravation and sleep quality has additionally been displayed in sound people. There was a connection between low CD4 levels $(<200$ cells/ $\mu \mathrm{L})$ and helpless sleep quality. Saberri et al, connecting drug use with helpless sleep quality might be an appearance of the symptoms of the HIV infection and unlawful substance use on the CNS (Saberi et al., 2011).

\section{Types of Sleep Disorders}

In certain examinations, sleep aggravations that happen in individuals with HIV, are assessed to happen as much as $70 \%$. In a review led by Kunisaki et al in regards to sleep issues in HIV, it was accounted for that sleep deprivation was more normal in HIV patients, the present circumstance was connected with the personal satisfaction and deteriorating of the ailment of patients who were regularly undiscovered (Kunisaki et al., 2021). Over $20 \%$ of individuals living with HIV met the survey measures for a sleeping disorder, which was related with fundamentally more awful phy sical, mental, and sleep quality. Individuals with HIV are viewed as multiple times bound to encounter a sleeping disorder than sound individuals, yet not many report past conclusion and treatment of sleep deprivation. Sleepless legs disorder and sleep apnea have been accounted for less oftentimes than sleep deprivation (Ren et al., 2018).

In a review directed by Gamaldo et al it was accounted for that $56 \%$ of HIV patients were determined to have clinical a sleeping disorder. Of the $25 \mathrm{HIV}$ victims, one individual was found to have parasomnia (sleep fear), two were found to have a circadian sleep musicality problem, two were found to have Sleepless Legs Syndrome (RLS), and 4 were found to have helpless sleep cleanliness (Gamaldo et al., 2013).

\section{a. Insomnia}

Insomnia is the most widely recognized sleep issue in the public arena. Objections that emerge fluctuate. As indicated by the International Classification of Sleep Disorders (ICSD)- 3, insomnia is an abstract view of the trouble in starting sleep, the term, combination, or nature of sleep, despite the fact that the victim is given sufficient opportunity to sleep. The frequency of sleep deprivation in individuals matured more than 15 years in Canada is 3.3 million individuals or one of 
every seven Canadians. In America 60 million individuals experience insomnia and a big part of patients in clinics whine of sleep deprivation. Ongoing sleep deprivation is frequently brought about by changes in circadian rhythms, every day conduct, natural variables, other sleep problems, development issues, clinical sicknesses, neurological issues, mental issues, period, pregnancy, drug utilized, drug reliance and different grumblings (Kunisaki et al., 2021)

Patients with HIV/AIDS experience protests of sleep deprivation are higher than everyone as indicated by past investigations. The majority of the exploration on insomnia in patients with HIV/AIDS has zeroed in on psychosocial factors. The presence of constant illness conditions can set off intense a sleeping disorder, which then, at that point, turns into an ongoing condition that goes with HIV disease. HIV indications, including agony and despondency, can deteriorate a sleeping disorder. Drug use is regularly connected with sleep unsettling influences, and the antiretroviral drug generally normally connected with sleep deprivation is the primary line non-nucleoside transcriptase inhibitor efavirenz, albeit these secondary effects frequently vanish following 3 months of utilization (Wu et al., 2015).

At present there are no rules for the treatment of sleep deprivation in individuals with HIV/AIDS (Wu et al., 2015). Sleep cleanliness is a prescribed treatment and simple to execute in essential consideration with the ideal impact of diminishing practices that can influence sleep or prompt an individual to be effectively alert, where the patient is approached to make a timetable. sleep wake routinely, do standard exercise, head to sleep just when truly feel tired, keep away from weighty dinners before sleep time, stay away from espresso, liquor and nicotine, make the room air faint and calm, keep away from sleeps, stay away from devices gadgets that produce splendid light like cellphones and others (Gutierrez et al., 2019).

Mental conduct treatment for insomnia (CBT-I) is the current norm of training for the primary line treatment of sleep deprivation. CBT-I incorporates a few parts: Sleep Sleepriction Therapy, Stimulus Control Therapy, Sleep Hygiene, and Cognitive Therapy (Wu et al., 2015).

Pharmacological treatments utilized for sleep deprivation incorporate benzodiazepines, benzodiazepine receptor agonists (BzRA), and antidepressants. Benzodiazepine treatment has been utilized rarely in view of its generally long halflife and its relentless morning impact (sedation) and potential for physical and mental reliance. BzRA has been demonstrated to be exceptionally compelling in treating a sleeping disorder, has a lower hazard of misuse and reliance than benzodiazepines, and has a short to medium half-life, so it seldom causes morning sedation. The high pervasiveness of substance maltreatment in patients with HIV should be viewed as while recommending benzodiazepines and BzRA (Monti, 2011; Wu et al., 2015)

\section{b. Obstructive sleep apnea syndrome}

Obstructive sleep apnea condition (OSAS) is a sleep issue that is very normal, however isn't well known by the layman or clinical local area in Indonesia. OSAS is the most widely recognized instance of sleep scattered relaxing. This illness has a wide effect and undermines the personal satisfaction. OSAS is related with an expanded danger of street auto collisions, as well as cardiovascular dreariness and mortality. A few epidemiological examinations in Asia show the commonness of OSAS is more normal in men than ladies. Conceivable danger factors that assume a part are craniofacial structures that tight the upper aviation route (Mete et al., 2013).

OSA is a sleep issue described by incomplete or complete blockage of the aviation routes that causes apnea and hypopnea during sleep. Apnea and hypopnea are trailed by oxygen desaturation and typically finishes with a short excitement. The danger of OSAS is expanded by a few elements. Antiretroviral drug treatment (ARV) can set off lipodystrophy where there is fat aggregation in the upper aviation route which will cause sleepricting and will generally close when the muscles unwind, particularly during the REM sleep stage. Patients with HIV/AIDS can likewise encounter weight gain overall because of diminished active work or symptoms of medicine that will prompt fixation. Provocative conditions related with HIV infection might build the danger of OSAS (Taibi, 2013)

Treatment of OSAS in patients with HIV/AIDS is vital to diminish side effects and fosleepall cardiovascular difficulties because of hypoxia, hypercapnia, and irritation related endothelial brokenness. Considering that patients with HIV/AIDS have an expanded danger of cardiovascular demise, treatment of OSAS to fosleepall cardiovascular entanglements is basic (Wu et al., 2015).

OSA requires long haul multidisciplinary the board. Therapy choices incorporate clinical, social and careful treatment. The current best treatment for OSA is nasal CPAP (Continuous positive aviation route pressure), both for gentle, moderate and extreme OSA. Elective treatment is acclimated to the seriousness of OSA, physical irregularities, hazard elements, and patient longings (Saberi et al., 2013; Wu et al., 2015)

Different medicines are accessible, albeit these are less viable and will more often than not be prescribed uniquely for 
gentle to direct OSAS. Oral machines can keep the upper aviation routes open during sleep by broadening the aviation routes by decreasing upper aviation route foldability (further develops muscle tone). In the condition that heftiness is found, conduct treatment should be possible where weight reduction, work out, positional treatment and staying away from liquor and narcotic medications before sleep time should be possible (Gutierrez et al., 2019)

There is evidence that untreated patients with severe OSA can develop fatal complications. Patients who do not receive therapy will have an increased incidence of fatal and non-fatal cardiovascular disorders, compared to the use of CPAP. One study reported higher mortality after 5 years in patients not using CPAP than those using CPAP (Taibi, 2013)

\section{c. Parasomnia}

Parasomnia is a condition or occasion that happens with arousing from sleep toward the start of sleep or during sleep that doesn't change the quality or amount of sleep. Parasomnias are strange practices that happen during sleep or the progress to sleep or during sleep. Parasomnias comprise of strange sleep related developments, practices, feelings, insights, dreams, and autonomic sensory system capacities. The commonness of parasomnias in America is higher at a youthful age than at an advanced age and the most well-known sort is sleep strolling. Announced more in men than ladies. The reasons for parasomnias incorporate hereditary qualities, mental factors like uneasiness, culpability, and pressure. Utilization of medications and substances that have results of sleep aggravations, these incorporate pain relieving drugs (narcotics, nonnarcotics, and adjuvants), antidepressants, caffeine, liquor and nicotine (Abdu and Dule, 2020).

Parasomnias are for the most part partitioned into two sorts, REM (Rapid Eye Movement) parasomnias and NREM (Non-Rapid Eye Movement) parasomnias. A review from Gamaldo et al, detailed that one HIV patient had a sleep problem parasomnia as sleep fear. Victims will feel sleepless, regularly with the conviction that there is an inevitable danger, joined by conduct that needs to run or make due. The term of sleep fear is around 30 seconds to 5 minutes and generally happens during the third of the evening (the mid an hour and a half of sleep) or during the beginning stage of sleep. Guideline of sleep and wake cycles and counteraction of lack of sleep can decrease the recurrence of event. Three methodologies are required, specifically adjustment of setting off or setting off factors, expanding the security of the sleep climate, and pharmacotherapy (Gamaldo et al., 2013).

\section{d. Sleep Releated Movement Disordered}

This diagnosis sort of sleep problem should be possible on the off chance that the patient accompanies grumblings of sleep deprivation, over the top daytime weariness, or non-sleeporative sleep brought about by developments during sleep or before sleep beginning. Developments that happen characteristically or as monopacic development issues and are straightforward in structure. Gamaldo et al, announced the frequency of Sleepless leg disorder (RLS) in two patients with HIV. This condition is a sleep related development problem that essentially affects personal satisfaction. RLS is a neurological sickness portrayed by a powerful desire or need to move the appendages, by and large connected with undesirable appendage impressions that deteriorate during sleep and improve with moving the legs or fingers or getting up. The predominance of RLS in everyone is assessed to be roughly $10 \%$. RLS order in light of the reason, in particular essential hereditary related and optional emerging from specific illnesses, way of life (caffeine, nicotine, liquor), drugs (SSRIs, tricyclic antidepressants, metoclopramide, prochlorperazine, dopamine adversaries, diphenhydramine), pregnancy, persistent kidney sickness with hemodialysis, family background of RLS, iron lack frailty, normal blood benefactor (Happe et al., 2007).

In a concentrate by Happe et al it was accounted for that HIV-tainted patients showed a fundamentally higher pervasiveness rate for RLS than everyone. HIV disease itself with immunologic changes and focal sensory system contribution might incline toward the danger of RLS in HIV-tainted patients. Since HIV contamination is an immunological issue with an expanded occurrence of fringe neuropathy, the commonness of RLS in this gathering of patients might be expanding. Also, patients with HIV contamination show weakened basal ganglia work at the beginning of disease. This may likewise add to the higher rate of RLS in HIV contamination (Happe et al., 2007).

\section{d. Circadian rhythm sleep disorders}

Circadian sleep musicality unsettling influences are irregularities in sleep beginning, sleep length and additionally inconsistency in the sleep wake cycle comparative with the day-night cycle. Circadian musicality sleep problems include industrious or repeating changes between circadian rhythms and sleep. Patients with this issue can not sleep at sleep time as 
a matter of fact. The sleep wake cycle is out of sync with circadian planning. Accordingly, patients grumble of a sleeping disorder or extreme drowsiness (Abdu and Dule, 2020; Taibi, 2013).

The HIV transcriptional trans-activator protein (Tat), which is let out of HIV-contaminated glial cells and macrophages in the cerebrum, assumes a significant part in HIV replication. HIV Tat is known to straightforwardly influence the circadian pacemaker situated in the suprachiasmatic core of the nerve center, hidden lentiviral circadian musicality brokenness. In a review directed by Wang et al, it was accounted for that the HIV Tat protein influences the circadian cadence by upsetting the circadian framework in HIV/AIDS patients and hence expanding the pace of melatonin discharge. High melatonin values related with the Tat protein might neutralize HIV-related helpless sleep quality during the advancement of HIV contamination (Taibi, 2013).

\section{Conclusion}

Combination of vitamin $\mathrm{E}$ in topical olive oil with metamizole decrease in PAI-1 levels in peritoneal fluid, macroscopic and microscopic degree of adhesion. This research is recommended for further experimental animal studies with other doses and methods of administration in the hope of obtaining better results so that their use is expected to be used as a practical application in surgical procedures.

\section{Competing intesleeps}

No competing intesleeps were disclosed.

\section{Conflict of intesleep}

The authors declare no conflict of intesleep, financial or otherwise.

\section{Acknowledgments}

declared none.

\section{References}

Abdu, Z., Dule, A., 2020. Poor quality of sleep among HIV-positive persons in Ethiopia. HIV/AIDS - Res. Palliat. Care 12, 621-628. https://doi.org/10.2147/HIV.S279372

Alikhani, M., Ebrahimi, A., Farnia, V., Khazaie, H., Radmehr, F., Mohamadi, E., Davarinejad, O., Dürsteler, K., Sadeghi Bahmani, D., Brand, S., 2020. Effects of treatment of sleep disorders on sleep, psychological and cognitive functioning and biomarkers in individuals with HIV/AIDS and under methadone maintenance therapy. J. Psychiatr. Res. 130, 260-272. https://doi.org/10.1016/j.jpsychires.2020.07.043

Bandera, A., Taramasso, L., Bozzi, G., Muscatello, A., Robinson, J.A., Burdo, T.H., Gori, A., 2019. HIV-associated neurocognitive impairment in the modern ART era: Are we close to discovering reliable biomarkers in the setting of virological suppression? Front. Aging Neurosci. 10. https://doi.org/10.3389/fnagi.2019.00187

Gamaldo, C.E., Spira, A.P., Hock, R.S., Salas, R.E., McArthur, J.C., David, P.M., Mbeo, G., Smith, M.T., 2013. Sleep, function and HIV: A multi-method assessment. AIDS Behav. 17, 2808-2815. https://doi.org/10.1007/s10461-0120401-0

Gutierrez, J., Tedaldi, E.M., Armon, C., Patel, V., Hart, R., Buchacz, K., 2019. Sleep disturbances in HIV-infected patients associated with depression and high risk of obstructive sleep apnea. SAGE Open Med. 7, 205031211984226. https://doi.org/10.1177/2050312119842268

Handayani, S., Ratnasari, N.Y., Husna, P.H., Marni, Susanto, T., 2019. Quality of Life People Living with HIV/AIDS and Its Characteristic from a VCT Centre in Indonesia. Ethiop. J. Health Sci. 29, 759-766. https://doi.org/10.4314/ejhs.v29i6.13

Happe, S., Kundmüller, L., Reichelt, D., Husstedt, I.W., Evers, S., 2007. Comorbidity of sleepless legs syndrome and HIV infection. J. Neurol. 254, 1401-1406. https://doi.org/10.1007/s00415-007-0563-2

Indonesian Ministry of Health, 2020. Laporan perkembangan HIV AIDS \& penyakit infeksi menular seksual (PIMS) triwulan II tahun 2020 (report of HIV AIDS and sexually transmitted infection trimester II year 2020). Kementeri. Kesehat. RI 4247608.

Kunisaki, K.M., De Francesco, D., Sabin, C.A., Winston, A., Mallon, P.W.G., Anderson, J., Bagkeris, E., Boffito, M., Doyle, N., Haddow, L., Post, F.A., Sachikonye, M., Vera, J., Khalil, W., Redline, S., 2021. Sleep Disorders in Human Immunodeficiency Virus: A Substudy of the Pharmacokinetics and Clinical Observations in People over Fifty (POPPY) Study. Open Forum Infect. Dis. 8, 1-9. https://doi.org/10.1093/ofid/ofaa561

Mete, T., Yalcin, Y., Berker, D., Ciftci, B., Guven, S.F., Topaloglu, O., Yavuz, H.C., Guler, S., 2013. Obstructive sleep 
apnea syndrome and its association with vitamin D deficiency. J. Endocrinol. Invest. 36, 681-685. https://doi.org/10.3275/8923

Oshinaike, O., Akinbami, A., Ojelabi, O., Dada, A., Dosunmu, A., John Olabode, S., 2014. Quality of sleep in an HIV population on antiretroviral therapy at an urban tertiary centre in Lagos, Nigeria. Neurol. Res. Int. 2014. https://doi.org/10.1155/2014/298703

Saberi, P., Comfort, M., Sheon, N., Johnson, M.O., 2013. Qualitative study of the quality of sleep in marginalized individuals living with HIV. Patient Prefer. Adherence 7, 499-507. https://doi.org/10.2147/PPA.S44595

Saberi, P., Neilands, T.B., Johnson, M.O., 2011. Quality of sleep: Associations with antiretroviral nonadherence. AIDS Patient Care STDS 25, 517-524. https://doi.org/10.1089/apc.2010.0375

Schank, M., Zhao, J., Moorman, J.P., Yao, Z.Q., 2021. The impact of hiv-and art-induced mitochondrial dysfunction in cellular senescence and aging. Cells 10,1-21. https://doi.org/10.3390/cells10010174

Taibi, D.M., 2013. Sleep Disturbances in Persons Living With HIV. J. Assoc. Nurses AIDS Care 24, S72-S85. https://doi.org/10.1016/j.jana.2012.10.006

Wu, J., Wu, H., Lu, C., Guo, L., Li, P., 2015. Self-reported sleep disturbances in HIV-infected people: A meta-analysis of prevalence and moderators. Sleep Med. 16, 901-907. https://doi.org/10.1016/j.sleep.2015.03.027 